Background There are major concerns and uncertainty regarding a possible reduction in growth velocity and final height of children with asthma who are long-term users of inhaled corticosteroids (ICS). We aimed to evaluate the association between ICS use of $>12$ months and growth.

Methods We initially searched MEDLINE and EMBASE in July 2013, followed by a PubMed search updated to June 2014. We used a combination of search terms involving drug names and adverse effects of interest (such as growth or height), and we also hand-searched reference lists of existing systematic reviews and trial reports. We selected RCTs and controlled observational studies of any ICS vs non-ICS control treatment in patients with asthma (treatment duration of at least 52 weeks). Meta-analysis of continuous outcomes (growth velocity in $\mathrm{cm} /$ year or final height in $\mathrm{cm}$ ) was conducted using RevMan 5.3. We analysed mean differences using inverse variance method, random effects model. Heterogeneity was assessed using the I2 statistic.

Results We found 21 relevant studies (seventeen RCTs and four observational studies) after screening 1876 hits from the search. Meta-analysis of 16 RCTs showed a significant association between ICS use and reduction in growth velocity compared to controls (pooled Mean Difference $-0.35 \mathrm{~cm} /$ year, 95\% CI -0.54 to -0.18 ). No significant reduction in growth velocity with ICS was reported in two observational studies of lower quality (pooled Mean Difference $0.03 \mathrm{~cm} /$ year, 95\% CI -0.61 to 0.67 ). Analysis of final adult height showed a mean reduction of -1.20 cm $(95 \%$ CI $-1.90 \mathrm{~cm}$ to $-0.50 \mathrm{~cm})$ with budesonide versus placebo in a high quality RCT. Meta-analysis of two lower quality observational studies found a non-statistically significant pooled mean reduction in final adult height of $-0.85 \mathrm{~cm}$ (95\% CI -3.35 to 1.65 ).

Conclusion Use of ICS for 12 months or more in children with asthma has a limited impact on annual growth velocity, with a slight reduction in final adult height. When interpreted in the context of the typical final adult height in the UK, ICS users may experience less than $0.7 \%$ reduction in height compared to non-ICS users.

\section{P235 PREDNISOLONE/CORTISOL SPOT TEST OF NON- ADHERENCE IN CORTICOSTEROID-DEPENDENT ASTHMA}

Adel Mansur. Heartlands Hospital, Birmingham, UK

10.1136/thoraxjnl-2014-206260.363

Background About 40\% of severe asthmatics require maintenance oral corticosteroids (OCS) for disease control. However, significant proportion of these patients continues to have poor disease control due to OCS unresponsiveness or non-adherence (Gamble 2009).

Methods Using a validated in-house developed liquid chromatography/mass tandem spectrometry (LQ/MSMS), we conducted spot measurement of serum prednisolone, prednisone and cortisol in 111 patients attending our severe asthma clinic over a 12 months period. Patients not on maintenance OCS comprised the control group. Suppressed cortisol (20nmol/l, were considered as compatible with adherence to OCS, whilst unsuppressed cortisol and undetectable prednisolone were considered as non-adherent. For validation purposes the test was repeated multiple times in few cases.

Results The prednisolone/cortisol spot test was conducted on 111 patients (79\% females) with $44(40 \%)$ were not on regular OCS (control group) and 67 (60\%) on maintenance OCS. The

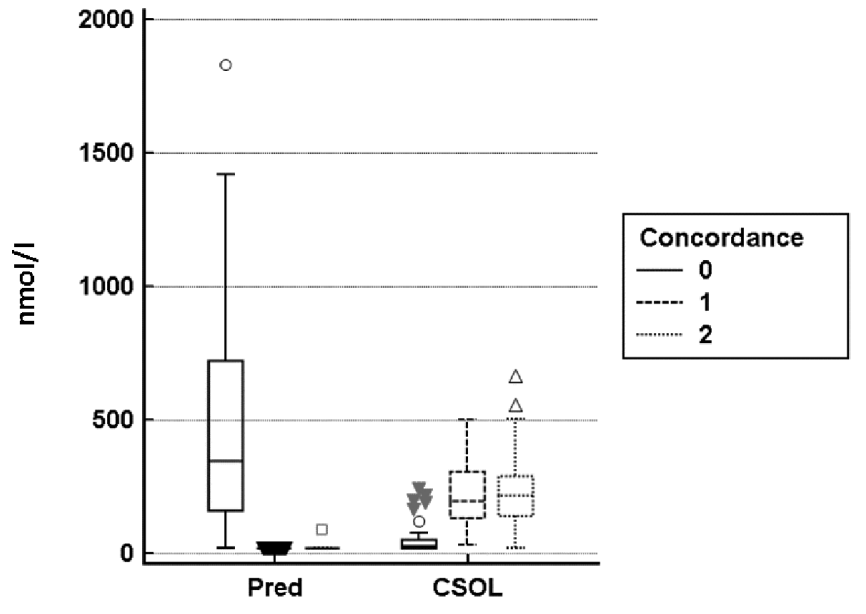

$0=$ concordant group, $1=$ non concordant group, and 2 non-OCS control Pred= Prednisolone, $\mathrm{CSOL}=$ cortisol

\section{Abstract P235 Figure 1}

spot test revealed non-adherence in 27/67 (40\%) of patients and adherence in 40/67 (60\%) of patients. The prednisolone/prednisone/cortisol assays were similar in non-adherent group and nonOCS group (figure). The mean daily prednisolone dose was $16.3,20.1$, and $0.0 \mathrm{mgs}$ in the adherent, non-adherent and nonOCS groups respectively. Non-adherent patients had lower BMI, and higher exacerbations frequency, blood eosinophil count, and fraction exhaled nitric oxide than OCS adherent group. The non-adherent group resembled more the non-OCS group with regard to aforementioned parameters.

We conclude that this prednisolone/cortisol spot test is reproducible and diagnostic of non-adherence to OCS in $40 \%$ of patients on maintenance OCS, and should be routinely measured in severe asthma clinics to improve patients management.

\section{P236 RELATIONSHIP BETWEEN BONE MINERAL DENSITY AND BONE TURNOVER MARKERS IN SEVERE ASTHMA PATIENTS ON SYSTEMIC CORTICOSTEROIDS}

${ }^{1}$ HGT Brice, ${ }^{1}$ JP Farrant, ${ }^{1} \mathrm{SJ}$ Fowler, ${ }^{2} \mathrm{U}$ Holmes, ${ }^{3} \mathrm{~J}$ Tang, ${ }^{3} \mathrm{C}$ Washbourne, ${ }^{3} \mathrm{I}$ Piec, ${ }^{3} \mathrm{~W}$ Fraser, ${ }^{4} \mathrm{~J}$ Sweeney, ${ }^{4} \mathrm{LG}$ Heaney, ${ }^{2} \mathrm{RM}$ Niven. ${ }^{1}$ University of Manchester, Manchester, Greater Manchester, ' 2 University Hospital of South Manchester, Manchester, Greater Manchester; ${ }^{3}$ University of East Anglia, Norwich, Norfolk; ${ }^{4}$ Belfast City Hospital, Belfast, Antrim

\subsection{6/thoraxinl-2014-206260.364}

Objectives Measure bone turnover markers (BTM) CTx, P1NP and bone-specific alkaline phosphatase (BsALP) in a severe asthma population using systemic corticosteroids (SCS). Assess bone mineral density (BMD) change in regional severe asthma clinics.

Background Severe asthma often requires regular SCS use. SCS cause several adverse effects including reduced bone metabolism; resorption is increased and formation is decreased resulting in osteoporosis. DXA scans monitor BMD in the hip and spine every 3-5 years. BMD decrease is treated with bone sparing medication (BSM), but treatment is retrospective and response takes years to assess. BTM represent markers of systemic bone metabolism and may offer a more efficient alternative. CTx is a resorption marker, P1NP and BsALP are formation markers.

Method Patients attending Manchester or Belfast severe asthma clinic with two or more DXA scans were identified from case files. We measured BTM in Manchester severe asthma patients 
and a control group of Manchester severe asthma patients requiring less than 3 courses of steroids per year. Using case files we collected data including DXA scan results, SCS use and osteoporosis risk factors.

Results BMD change and BTM did not correlate overall (patients $\mathrm{n}=78$, controls $\mathrm{n}=18$ ) but correlated negatively in patients where the most recent DXA was

Conclusion This study has confirmed that BTM may be potentially used as a surrogate for BMD measurement; however a more a prospective study where BTM are measured at the time of two DXA scans which are years apart is required.

\section{P237 A REVIEW OF THE STEROID SPARING IMPACT OF MYCOPHENOLATE MOFETIL IN THE SEVERE ASTHMA POPULATION AT THE NORTH WEST LUNG CENTRE, UNIVERSITY HOSPITAL SOUTH MANCHESTER}

${ }^{1} \mathrm{KE}$ George, ${ }^{1} \mathrm{HGT}$ Brice, ${ }^{1} \mathrm{SJ}$ Fowler, ${ }^{2} \mathrm{U}$ Holmes, ${ }^{2} \mathrm{R}$ Daly, ${ }^{2} \mathrm{RM}$ Niven. ${ }^{1}$ University of Manchester, Manchester, Greater Manchester; ${ }^{2}$ University Hospital South Manchester, Manchester, Greater Manchester

\subsection{6/thoraxjn-2014-206260.365}

Objectives To determine whether Mycophenolate mofetil (MMF) is an effective steroid sparing agent in a single centre cohort of severe asthma patients.

Background MMF is a powerful inhibitor of purine and pyrimidine synthesis via the de novo pathway, upon which lymphocyte production is dependent. It is currently licensed for use in transplant rejection prophylaxis. Other immunosuppressant therapies have been used off licence in difficult-to-treat asthma patients under specialist supervision in an effort to reduce corticosteroid use. MMF is currently a third line immunosuppressant after methotrexate and azathioprine at the North West Lung Centre. Methods A retrospective data analysis was performed including all patients under specialist asthma care at UHSM that were previously or currently treated with MMF. Annualised average daily steroid dose was calculated from the available data in patient case notes. This was calculated for 12 months prior to

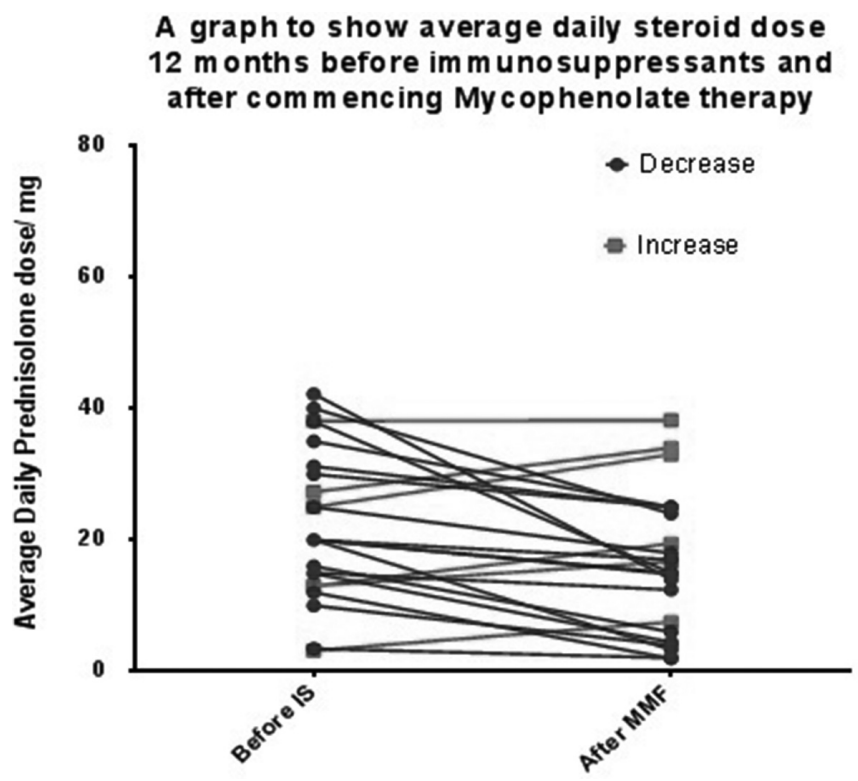

Abstract P237 Figure 1 commencing any immunosuppressant therapy and during MMF treatment. Exacerbation and hospital admission rates were also recorded.

Results A total of 34 patients were identified as being on MMF for severe asthma for at least 8 weeks. 11 did not tolerate MMF or had no response and subsequently stopped. The primary analysis was carried out on 23 patients and a secondary post hoc analysis was performed on all patients who had been on treatment for a minimumof 6 months at the time of the study $(\mathrm{N}=12)$. The average yearly steroid sparing impact of MMF was $5.9 \mathrm{mg}$ per day, $(\mathrm{p}<0.005) .74 \%$ had an overall reduction and $35 \%$ achieved a reduction of $10 \mathrm{mg}$ or more. This value was lower in those who had been on treatment for $>6$ months $(\Delta$ $3.9 \mathrm{p}=0.20$ ). There was no statistically significant reduction in admission or exacerbation rates.

Conclusion MMF has shown a small steroid sparing effect in this retrospective analysis, although the effect appeared less positive in the sub-group of those analysed after being on treatment for at least 6 months further analysis of the potential benefits of MMF in this patient population is required.

\section{P238 THE EFFECT OF INHALATION DURATION ON LUNG DEPOSITION WITH A PRESSURISED METERED-DOSE INHALER (PMDI)}

${ }^{1} \mathrm{C}$ Van Holsbeke, ${ }^{2} \mathrm{~J}$ Marshall, ${ }^{1} \mathrm{~J}$ De Backer, 'W Vos. ${ }^{1}$ Fluidda Nv, Kontich, Belgium; ${ }^{2}$ Mundipharma International, Cambridge, UK

10.1136/thoraxjnl-2014-206260.366

Rationale Although the guidance for using a pMDI is to inhale 'slow and deeply', many patients inhale fast over a short duration. The ERS/ISAM Task Force suggested 'slowly' equates to inhaling over 4-5 seconds (s) for adults1, a much clearer instruction. This study therefore examined the influence of inhalation time on total lung deposition (TLD) using Functional Respiratory Imaging (FRI).

Methods Three-dimensional airway models of 6 asthma patients (mean FEV1 83\%), treated with an ICS/LABA combination, were included. The lung deposition characteristics of an HFAbased pMDI (MMAD $\sim 3.0 \mu \mathrm{m}$; fine particle fraction (FPF) $\sim 40 \%$ ) were assessed using FRI. Simulations were performed on 3 different inhalation profiles matched for the same inspiratory volume $(3 \mathrm{~L})$ with durations of $1 \mathrm{~s}, 3 \mathrm{~s}$ and $5 \mathrm{~s}$ and actuation at start of inhalation.

Results For the $1 \mathrm{~s}, 3 \mathrm{~s}$ and $5 \mathrm{~s}$ profiles, the TLD values were $22.81 \pm 3.71 \%, 36.13 \pm 2.51 \%$ and $41.61 \pm 3.11 \%$ of nominal dose respectively, and were predicted using a concave down quadratic model $(\mathrm{R} 2=0.87, \mathrm{p}<0.001)$. The central to peripheral deposition ratios were $1.58,0.81$ and 0.57 respectively.

Conclusions A $5 \mathrm{~s}$ inhalation led to highest TLD with greatest peripheral deposition. Increased deposition with longer times mainly reflected increased peripheral deposition, central deposition was less affected by flow rate. These data support ERS/ ISAM guidance for inhaling over 4-5 sec to optimise deposition, although similar TLD were achieved with $3 \mathrm{~s}$. These data also suggest that high FPF pMDIs can achieve reasonable deposition even with short, fast inhalations.

\section{REFERENCES}

1 Laube BL, et al. ERJ 2011;37(6):1308-417

Disclaimer acknowledgement: Reproduced with permission of the European Respiratory Society: Eur Resp J September 2014; 44, Suppl. 58: In Press 